\title{
Acute odontogenic maxillary sinusitis treated endodontically - two case reports and 15-day CBCT outcome
}

Lauter Eston Pelepenko¹, Marina Canali Lângaro', Mateus Silveira Martins Hartmann ${ }^{1}$

Patients presented acute recurrences from Otorhinolaryngology treatments with unilateral symptoms compatible with maxillary sinusitis with a dental origin (MSDO). Cone beam computed tomography (CBCT) showed sinus extensive membrane thickening that was not visible at periapical exam associated to the maxillary molar infection in both cases. Respiratory structures assessment was possible using a 6-inches field of view CBCT. Non-surgical endodontic treatment (case 1) performed and retreatment (case 2) showed a fast symptoms relief. Recall examination after 15 days revealed healthy soft tissues with normal periodontal probing and no dental mobility. In addition, 15-day CBCTs revealed initial maxillary sinus membrane recovery after the endodontic approaches in the reported cases. No additional medical intervention nor antibiotics administration were necessary in these MSDO cases management. Long-term follow-up in such cases is advisable to exclude other potential dental or respiratory issues. Diagnose and follow-up using CBCT presented significant sinus membrane thick reduction with no symptom's recurrence after the endodontic treatment.

Keywords: Maxillary sinus. Apical periodontitis. Cone-Beam Computed Tomography. Root canal therapy. Maxillary sinusitis. Molar. 


\section{Introduction}

Chronic maxillary sinusitis is an inflammatory reaction of membrane covering maxillary sinus (MS). It is usually asymptomatic, but occasionally this condition shows acute signs and symptoms associated to a sinusitis crisis ${ }^{1}$. Maxillary sinusitis with a dental origin (MSDO) usually presents unilateral symptoms ${ }^{2}$ varying from a small discomfort to an acute sinus infection. Otorhinolaryngologists usually consider this maxillary condition to infectious or allergic diseases as main factors for sinus membrane symptomatic inflammation prescribing antibiotic therapies, drainage maxillary procedures or both. On the other hand, dental practitioners usually diagnose dental periapical alterations in endodontic image exams regardless MS evaluation ${ }^{3}$.

Proper MSDO diagnose and treatment must consider both patient respiratory complaints and maxillary posterior dental evaluation ${ }^{4}$. Close relation from posterior maxillary teeth roots to MS has been widely researched ${ }^{5}$. Normal mucosal MS membrane is $<1 \mathrm{~mm}$ in thickness and usually not discernible radiographically. Even in posterior dental roots relatively far from this anatomic structure, membrane thickening may be present as a result from an endodontic dental infection or periodontal disease ${ }^{6-10}$.

From the endodontic aspect, large periapical lesions have clear identification using two-dimensional exams such as periapical or panoramic radiograph. Initial infection in posterior maxillary teeth without a bone loss observable in these types of exams may not have proper identification ${ }^{11}$. Tridimensional exams such as cone beam computed tomography (CBCT) for planning such cases provides proper conditions to view each root individually and its relationship to MS floor ${ }^{8,9,11,12}$. In MSDO acute cases clinical examination and endodontic tests in association with dental image exams must recognize dental etiology for this respiratory condition ${ }^{13-15}$.

The objective of this cases report is to present two acute unilateral maxillary sinusitis cases with dental origin treated endodontically using CBCT evaluation for diagnose and treatment planning. Also, to show 15-day exam after endodontic therapy presenting a fast MS membrane response to nonsurgical endodontic treatment without antibiotics. In both cases no further medical support was necessary.

\section{Case Reports}

The Helsinki Declaration and Euratom SEDENTEXCT project were followed as guidelines in these cases management as justification and referral criteria for CBCT use. This exam should not be selected as first choice, unless a history and clinical examination have been performed. CBCT used as routine imaging is unacceptable practice.

\section{Case 1}

\section{Patient selection}

A 57-year-old woman related diffuse mild pain on left maxillary region as the chief complaint and could not identify the source. First approach proposed by Otorhinolaryngology specialist consisted in nasal irrigation and analgesic medication a month ago. Symptoms persistence led the patient to suspect a dental origin. 


\section{Dental Report and Examination}

Diagnostic periapical radiograph (D-speed film, Carestream Health, Rochester, NY) obtained using parallel object-film angle (ProDental 70 kVp Periapical, Ribeirão Preto, SP, Brazil) from region, showed a 15-year left maxillary second premolar endodontic treatment without periapical lesion (figure 01A). Pulp vitality assessed by cold test presented positive response for first premolar and second molar. First molar presented an altered response to cold test regardless apical apparent integrity observed in this exam. Pulp chamber reduction was evident in this tooth. Percussion test showed a similar normal response for all teeth tested. Periodontal probing measured 3-mm deep and no mobility observed.
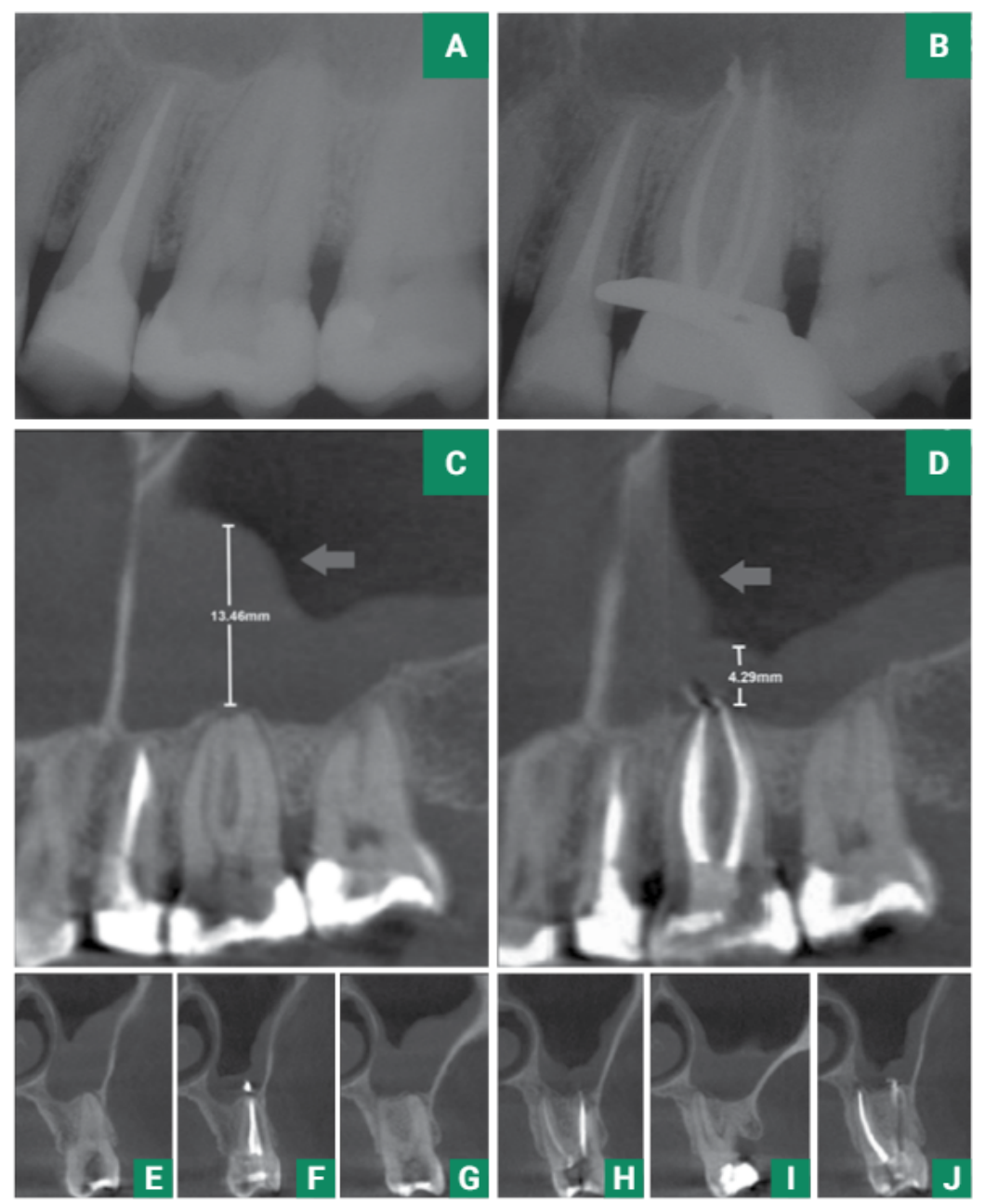

Figure 01. Case 1 images: (A) diagnostic periapical showing a 15-year-old endodontic treatment without apparent apical alteration (a CBCT was required), (B) left first molar endodontic obturation after root canal treatment, $(\mathrm{C})$ initial panoramic CBCT view showing considerable maxillary membrane thickening (arrow) associated to the acute unilateral symptoms, (D) 15-day after endodontic treatment CBCT view showing initial membrane recovery (arrow) and complete acute symptoms relief, (E) before endodontic treatment mesiobuccal root axial view showing maxillary membrane thickening and reduced air permeability, $(F)$ after endodontic treatment showing initial maxillary membrane recovery and complete symptoms relief. Same situation observed in distobuccal root before treatment $(\mathrm{G})$, distobuccal root after treatment $(\mathrm{H})$, palatal root before treatment $(\mathrm{I})$, and palatal root after treatment $(\mathrm{J})$. 
To evaluate the maxillary sinus relationship with the maxillary teeth, a CBCT (Op 300, Kavo Dental, Milwaukee, USA) with150 $\mathrm{mm}$ voxel size obtained from left maxillary region provided region tridimensional view ${ }^{14}$. CBCT was evaluated and first molar roots presented close relationship to the MS floor with cortical interruption and incipient apical periodontitis (figure $01 \mathrm{C}, \mathrm{E}, \mathrm{G}$ and I). In addition, exam showed a considerable MS membrane thickening. Clinical evaluation repeated six days after first approach, using now information obtained in CBCT exam, showed a negative response to cold on maxillary first molar and same result for all other tested teeth. Apical diagnosis established symptomatic apical periodontitis associated to MS acute symptoms.

\section{Nonsurgical Endodontic Treatment}

Negative cold test response obtained in the second evaluation, persistent apical percussion sensitivity and MS membrane thickening associated to the maxillary first molar indicated endodontic treatment necessity. Local infiltrative anesthesia technique used $1.8 \mathrm{~mL}$ mepivacaine chlorhydrate (Mepiadre $2 \%$ with epinephrine 1:100.000, DFL dental, Rio de Janeiro, RJ, Brazil). After rubber dam isolation, access cavity initiated using a 1016-round bur (KG Sorensen, Cotia, SP, Brazil) compatible to tooth crown size and completed with a 4083-cylindrical bur (KG Sorensen, Cotia, SP, Brazil). Access showed no visible dentin cracks.

Obtaining root canal access, tooth presented necrotic pulp with a scarce bleeding only on palatal root canal. In sequence, all four canals were scouted using Kerr 8 to 15-hand files ${ }^{16}$ (Dentsply Maillefer, Ballaigues, Switzerland). Root canal irrigation used a $5-\mathrm{mL}$ saline solution (LBS, Ultrafarma, SP, Brazil) per canal during preparation for debris removal using a $24-G$ needle (Nipro, Sorocaba, SP, Brazil) ${ }^{17}$ repeated between each instrument and hand file. Root canal initial flare was repeated three times using in-and-out pecking movements ${ }^{18,19}$ with reciprocating WaveOne Gold Small (Dentsply Sirona Endodontics, Ballaigues, Switzerland) using X-Smart Plus motor (XSM, Dentsply Maillefer, Switzerland) according to manufacturer's protocol. For working length (WL) determination, when Propex II electronic apex locator (Dentsply Maillefer, Ballaigues, Switzerland) read 'apex', defined WL measure ${ }^{20}$.

Irrigation during mechanical instrumentation used 2\% chlorhexidine gel (Natupharma, Passo Fundo, RS, Brazil) due its biocompatible and antimicrobial properties ${ }^{21-26}$. Root canal preparation used WaveOne Gold Small reaching full WL without apical pressure $^{27,28}$. Instrument debris removal after pecking movements used a sterile gauze. To complement apical root canal instrumentation, HERO 642 system (MicroMega, Besaçon, France) in rotary motion also without apical pressure ${ }^{29}$ dilated up to an ISO size 45/.02 diameter ${ }^{30}$ all root canals according to manufacturer's protocol. Except on second mesiobuccal canal (MB2) prepared up to an ISO size 35/.02 diameter, compatible to its internal reduced anatomy.

After instrumentation, passive ultrasonic irrigation (PUI) used ultrasonic E1 Irrisonic tip (Helse Ultrasonic Br, SP, Brazil) for 15 seconds per root canal positioned WL $2 \mathrm{~mm}$ short ${ }^{31}$ according to manufacturer's protocol using saline solution irrigation changed four times for each root canal. PUI used $80 \mathrm{~mL}$ saline solution in total. A chelating agent, 3 mL EDTA 17\% solution (Natupharma, Passo Fundo, RS, Brazil) irrigation ${ }^{23}$ and a $5 \mathrm{~mL}$ final saline solution prior to final obturation finished root canal preparation. 
Absorbent paper points ISO size 45 (Meta Biomed, Seoul, Korea) dried root canals. Once they presented dry, a single visit treatment ${ }^{32}$ completed root canal treatment.

Obturation used vertical hydraulic condensation gutta-percha cones FR-EL infinite taper cones (Odous de Deus, MG, Brazil) calibrated according to final apical diameter combined with AhPlus root canal sealer (Dentsply Maillefer, Ballaigues, Switzerland) (figure $01 \mathrm{~B}$ ). Immediate tooth restoration using direct resin composite avoided contamination. Proper oclusal adjustment and polishing finished restorative procedures. Postoperative instructions given did not include any antibiotics, just analgesic medication, if necessary.

\section{5-day Follow-up}

Pain gradually ceased after nonsurgical endodontic procedure. There was no other Otorhinolaryngology procedure immediately after root canal treatment. To evaluate immediate MS response, a 15-day CBCT analysis showed membrane initial recovery (figure $01 \mathrm{D}, \mathrm{F}, \mathrm{H}, \mathrm{J}$ ).

\section{Case 2}

\section{Patient selection}

A 28-year-old female related severe diffuse right earache as the chief complaint. Patient related history of 14-days antibiotic therapy proposed by Otorhinolaryngology specialist two months before, but symptoms acutely recurred. In addition, a professional ear cleaning attempted to solve similar pain related six months before.

\section{Dental Report and Examination}

Dental history included in maxillary arch only a 1-year old root canal treatment performed in right first molar (figure 02). Vitality cold test presented positive for all other right maxillary teeth, except for the first molar. Percussion test showed normal response including for first molar. To evaluate MS relation to molar roots a CBCT intentionally included both sides for air permeability comparison to asymptomatic left MS. Right MS cavity considerable air permeability reduction was observed when compared to left MS cavity. First molar nonsurgical endodontic retreatment was advised due to symptoms related by the patient, short endodontic filling observed in periapical radiograph in all three roots and apical radiolucency.

\section{Nonsurgical Endodontic Retreatment}

Endodontic retreatment used same local infiltrative anesthesia from previous reported clinical case. After cavity access, WaveOne gold primary (Dentsply Sirona Endodontics, Ballaigues, Switzerland) removed root canal filling according to manufacturer's protocol ${ }^{33}$. Between each instrument insertion, $5 \mathrm{~mL}$ saline solution removed filling material debris and $2 \%$ chlorhexidine gel served as lubricant agent ${ }^{25}$. WL determination used 'apex' reading as reference. Root canal filling removal did not use any gutta-percha solvents. Instrumentation used HERO 642 system up to an ISO size 45/.02 diameter cleaned after three pecking motions. Both clinically and in CBCT examination, MB2 was not visible. Final irrigation protocol, root canal filling and tooth resto- 

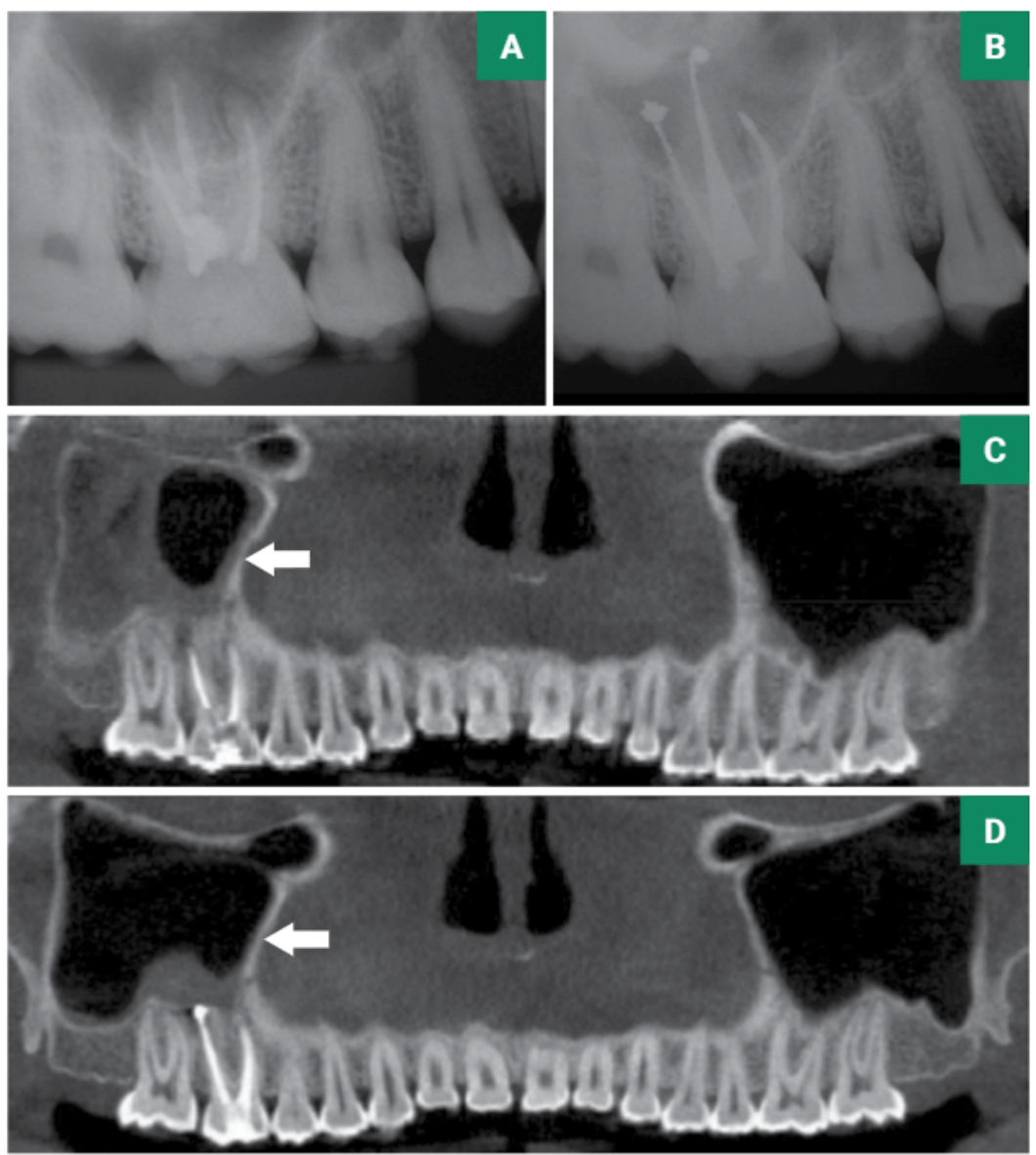

Figure 02. Case 2 images: (A) diagnostic periapical showing a 1-year-old endodontic treatment with apical radiolucency (a CBCT was required to the patient to evaluate the maxillary sinus air permeability and the first molar internal anatomy nominated to the endodontic retreatment), (B) right first molar endodontic obturation after root canal retreatment, (C) initial panoramic CBCT view showing considerable right maxillary sinus obliteration (arrow) associated to the earache acute symptoms, (D) 15-day after right molar endodontic retreatment CBCT view showing air permeability retrieval (arrow), initial membrane recovery and complete acute symptoms relief.

ration followed same procedures used in first case. Postoperative instructions did not included antibiotics. There was no other Otorhinolaryngology procedure after nonsurgical root canal retreatment.

\section{5-day Follow-up}

Acute pain related gradually reduced without any antibiotic medication. As response to endodontic retreatment, CBCT analysis 15-day after showed MS membrane recovery and air permeability comparable to left unaffected side (figure 02).

\section{Discussion}

The most common cause of rhinosinusitis is viral, inducing bacterial secondary respiratory infections. This condition is usually treated with decongestants and sometimes 
antibiotics but can occasionally be therapy-resistant or recurrent ${ }^{15}$. In such cases, radiologic examination is often required to exclude an alternative etiology or to facilitate other treatment options ${ }^{1,15}$. In both cases reported, initial treatments showed symptoms recurrence demanding additional investigation which included detailed dental examination. Particularly in the second case, treated molar was the only dental procedure in all maxillary arch. Apical periodontitis association to MS condition could be observed.

The relationship between dental roots and $\mathrm{MS}$ is determined using CBCT exam ${ }^{5,9,13}$. Incipient periapical lesions are also properly diagnosed using this exam. Periapical radiograph has less sensitivity to show periapical alterations when cortical bone plates remains unaltered ${ }^{11}$. This fact justifies CBCT analysis when MSDO is suspected, especially with unilateral symptoms such as in the described cases ${ }^{2-4}$. As observed in both cases, odontogenic sinusitis was recurrent to medical therapy and required dental disease treatments. When MSDO is properly diagnosed, usually dental treatment alone is adequate to solve odontogenic sinusitis without antibiotics ${ }^{1}$.

According to American College of Physicians ${ }^{34}$ clinicians should reserve antibiotic treatment for acute bilateral bacterial rhinosinusitis in patients with persistent symptoms for more than 10 days, high fever and purulent nasal discharge. None of our cases presented such symptoms, especially fever. Unilateral symptoms related by both patients indicated dental origin should be investigated ${ }^{8}$. Conventional 7 to 15 -day antibiotic treatments often temporarily diminish acute symptoms but recurrence is disturbing to the patient ${ }^{15}$. There is not available any case report showing MS membrane 15-day response to nonsurgical endodontic treatment or retreatment without antibiotics, same antibiotic therapy period used for maxillary sinusitis crisis.

A reported case showed acute symptoms relief shortly after root canal initial debridement ${ }^{35}$ based in panoramic and periapical exams in two appointments. Our cases showed similar response after this period in single visit treatments. Antimicrobial and biocompatible chlorhexidine gel properties used during root canal preparation was desirable in these cases due to intimate relationship from dental roots to MS structures ${ }^{22}$.

Another case series ${ }^{14}$ described 3-month MS membrane thickening recovery associated to chronic maxillary sinusitis, suggesting that fully resolved mucositis was not common 3 months after endodontic treatment. More studies regarding to time necessary to complete MS membrane recovery are necessary ${ }^{10}$, but differential diagnose must be done individually considering patient uniqueness, both dental and medical history.

Both cases involved maxillary first molars and have shown MSDO symptoms relief to a nonsurgical endodontic therapy until the moment. Initial otorhinolaryngologic approaches without considering dental origin were not successful in these cases. Complex internal anatomy usually showing additional root canals must be carefully scouted during endodontic treatment to completely remove any potential infection source that could affect MS. CBCT exams taken in both cases were useful to be familiar with root canal internal anatomy in advance 9 . A 15-day exam is not widely requested due to additional radiation exposure. In these cases, they were necessary to ensure MS air permeability retake after endodontic therapies. The immediate nonresponsive outcome only to endodontic therapy would demand additional otorhinolar- 
yngologic procedures, but that was not necessary in these cases. A long-term clinical follow-up in such cases is important and should be advised to patient.

In conclusion, both case reports have shown MSDO symptoms relief to a nonsurgical endodontic therapy until the moment. Initial otorhinolaryngologic approaches without considering dental origin were not successful to these cases. Within this study limitations, CBCT imaging provided necessary information to diagnose, treat and evaluate immediate outcome in MSDO cases. Communication between Radiologists, Endodontists and Otorhinolaryngologists is necessary to diagnose MSDO cases and propose adequate treatment.

\section{Acknowledgments}

The authors deny any conflicts of interest related to this study. We would also like to thank Dr. Volmir João Fornari and Dr. José Roberto Vanni scientific and clinical consultants.

\section{References}

1. Patel NA, Ferguson BJ. Odontogenic sinusitis: an ancient but under-appreciated cause of maxillary sinusitis. Curr Opin Otolaryngol Head Neck Surg. 2012 Feb;20(1):24-8. doi: 10.1097/ M00.0b013e32834e62ed.

2. Pokorny A, Tataryn R. Clinical and radiologic findings in a case series of maxillary sinusitis of dental origin. Int Forum Allergy Rhinol. 2013 Dec,3(12):973-9. doi: 10.1002/alr.21212.

3. Mehra P, Jeong D. Maxillary sinusitis of odontogenic origin. Curr Infect Dis Rep. 2008 May, 10(3):205-10

4. Workman AD, Granquist EJ, Adappa ND. Odontogenic sinusitis: developments in diagnosis, microbiology, and treatment. Curr Opin Otolaryngol Head Neck Surg. 2018 Feb;26(1):27-33. doi: 10.1097/M00.0000000000000430.

5. Kwak HH, Park HD, Yoon HR, Kang MK, Koh KS, Kim HJ. Topographic anatomy of the inferior wall of the maxillary sinus in Koreans. Int J Oral Maxillofac Surg. 2004 Jun;33(4):382-8.

6. Bauer W. Maxillary sinusitis of dental origin. Am J Orthod Oral Surg. 1943 Mar,29(3):B133-51.

7. Hauman $\mathrm{CH}$, Chandler NP, Tong DC. Endodontic implications of the maxillary sinus: a review. Int Endod J. 2002 Feb;35(2):127-41.

8. Lu Y, Liu Z, Zhang L, Zhou X, Zheng Q, Duan X, et al. Associations between maxillary sinus mucosal thickening and apical periodontitis using cone-beam computed tomography scanning: a retrospective study. J Endod. 2012 Aug;38(8):1069-74. doi: 10.1016/j.joen.2012.04.027.

9. Pagin O, Centurion BS, Rubira-Bullen IR, Alvares Capelozza AL. Maxillary sinus and posterior teeth: accessing close relationship by cone-beam computed tomographic scanning in a Brazilian population. J Endod. 2013 Jun;39(6):748-51. doi: 10.1016/j.joen.2013.01.014.

10. Kamburoğlu K, Yılmaz F, Gulsahi K, Gulen O, Gulsahi A. Change in periapical lesion and adjacent mucosal thickening dimensions one year after endodontic treatment: volumetric cone-beam computed tomography assessment. J Endod. 2017 Feb;43(2):218-224. doi: 10.1016/j.joen.2016.10.023.

11. Torabinejad M, Rice D, Maktabi O, Oyoyo U, Abramovitch K. Prevalence and size of periapical radiolucencies using cone-beam computed tomography in teeth without apparent intraoral radiographic lesions: a new periapical index with a clinical recommendation. J Endod. 2018 Mar;44(3):389-394. doi: 10.1016/j.joen.2017.11.015. 
12. Goodell K, Mines P, Kersten D. Impact of cone-beam computed tomography on treatment planning for external cervical resorption and a novel axial slice-based classification system. J Endod. 2018 Feb;44(2):239-244. doi: 10.1016/j.joen.2017.

13. Cymerman $\mathrm{JJ}$, Cymerman $\mathrm{DH}, \mathrm{O}$ 'Dwyer RS. Evaluation of odontogenic maxillary sinusitis using cone-beam computed tomography: three case reports. J Endod. 2011 Oct;37(10):1465-9. doi: 10.1016/j.joen.2011.06.015.

14. Nurbakhsh B, Friedman S, Kulkarni GV, Basrani B, Lam E. Resolution of maxillary sinus mucositis after endodontic treatment of maxillary teeth with apical periodontitis: a cone-beam computed tomography pilot study. J Endod. 2011 Nov,37(11):1504-11. doi: 10.1016/j.joen.2011.07.007.

15. Fredriksson MV, Öhman A, Flygare L, Tano K. When maxillary sinusitis does not heal: findings on CBCT scans of the sinuses with a particular focus on the occurrence of odontogenic causes of maxillary sinusitis. Laryngoscope Investig Otolaryngol. 2017 Dec 11;2(6):442-446. doi: 10.1002/lio2.130.

16. De-Deus G, Belladonna FG, Souza EM, Alves VO, Silva EJ, Rodrigues E, et al. Scouting ability of 4 pathfinding instruments in moderately curved molar canals. J Endod. 2016 Oct;42(10):1540-4. doi: 10.1016/j.joen.2016.07.001.

17. Nielsen BA, Baumgartner C. Comparison of the endovac system to needle irrigation of root canals. J Endod. 2007 May;33(5):611-5.

18. Amaral ROJF, Leonardi DP, Gabardo MCL, Coelho BS, Oliveira KV, Baratto-Filho F. Influence of cervical and apical enlargement associated with the Waveone system on the transportation and centralization of endodontic preparations. J Endod. 2016 Apr;42(4):626-31. doi: 10.1016/j.joen.2015.12.005.

19. Borges ÁH, Pereira TM, Porto AN, de Araújo Estrela CR, Miranda Pedro FL, Aranha AM, et al. The influence of cervical preflaring on the amount of apically extruded debris after root canal preparation using different instrumentation systems. J Endod. 2016 Mar;42(3):465-9. doi: 10.1016/j.joen.2015.10.010.

20. Üstün Y, Aslan T, Şekerci AE, Sağsen B. Evaluation of the reliability of cone-beam computed tomography scanning and electronic apex locator measurements in working length determination of teeth with large periapical lesions. J Endod. 2016 Sep;42(9):1334-7. doi: 10.1016/j.joen.2016.06.010.

21. Leonardo MR, Tanomaru Filho M, Silva LA, Nelson Filho P, Bonifácio KC, Ito IY. In Vivo antimicrobial activity of $2 \%$ chlorhexidine used as a root canal irrigating solution. J Endod. 1999 Mar;25(3):167-71.

22. Ferraz CC, Gomes BP, Zaia AA, Teixeira FB, Souza-Filho FJ. In Vitro Assessment of the antimicrobial action and the mechanical ability of chlorhexidine gel as na endodontic irrigant. J Endod. 2001 Jul;27(7):452-5.

23. Moreira DM, Almeida JF, Ferraz CC, Gomes BP, Line SR, Zaia AA. Structural analysis of bovine root dentin after use of different endodontics auxiliary chemical substances. J Endod. 2009 Jul;35(7):1023-7. doi: 10.1016/j.joen.2009.04.002.

24. Souza M, Cecchin D, Farina AP, Leite CE, Cruz FF, Pereira CC, et al. Evaluation of chlorhexidine substantivity on human dentin: a chemical analysis. J Endod. 2012 Sep;38(9):1249-52. doi: 10.1016/j.joen.2012.06.003.

25. Barbosa-Ribeiro M, De-Jesus-Soares A, Zaia AA, Ferraz CC, Almeida JF, Gomes BP. Quantification of lipoteichoic acid contents and cultivable bacteria at the different phases of the endodontic retreatment. J Endod. 2016 Apr,42(4):552-6. doi: 10.1016/j.joen.2016.01.002.

26. Zandi H, Rodrigues RC, Kristoffersen AK, Enersen M, Mdala I, Ørstavik D, et al. Antibacterial effectiveness of 2 root canal irrigants in root-filled teeth with infection: a randomized clinical trial. J Endod. 2016 Sep;42(9):1307-13. doi: 10.1016/j.joen.2016.06.006.

27. Zuolo ML, Carvalho MC, De-Deus G. Negotiability of second mesiobuccal canals in maxillary molars using a reciprocating system. J Endod. 2015 Nov;41(11):1913-7. doi: 10.1016/j.joen.2015.08.004. 
28. Bitencourt MA, Rocha DGP, Bueno CES. Incidence of dentinal defects on the external apical root surface after instrumentation with waveone reciprocating files at different working lengths. J Endod. 2017 Mar;43(3):491-495. doi: 10.1016/j.joen.2016.11.010.

29. Aksel H, Küçükkaya Eren S, Çakar A, Serper A, Özkuyumcu C, Azim AA. Effect of instrumentation techniques and preparation taper on apical extrusion of bacteria. J Endod. 2017 Jun;43(6):10081010. doi: 10.1016/j.joen.2017.01.014.

30. Sant'Anna Júnior A, Cavenago BC, Ordinola-Zapata R, De-Deus G, Bramante CM, Duarte MA. The effect of larger apical preparations in the danger zone of lower molars prepared using the Mtwo and Reciproc systems. J Endod. 2014 Nov;40(11):1855-9. doi: 10.1016/j.joen.2014.06.020.

31. Simezo AP, da Silveira Bueno CE, Cunha RS, Pelegrine RA, Rocha DG, de Martin AS, et al. Comparative analysis of dentinal erosion after passive ultrasonic irrigation versus irrigation with reciprocating activation: an environmental scanning electron study. J Endod. 2017 Jan;43(1):141-146. doi: 10.1016/j.joen.2016.09.016..

32. Moreira MS, Anuar ASN, Tedesco TK, Dos Santos M, Morimoto S. Endodontic treatment in single and multiple visits: an overview of systematic reviews. J Endod. 2017 Jun;43(6):864-870. doi: 10.1016/j.joen.2017.01.021.

33. Silva EJ, Sá L, Belladonna FG, Neves AA, Accorsi-Mendonça T, Vieira VT, et al. Reciprocating versus rotary systems for root filling removal: assessment of the apically extruded material. J Endod. 2014 Dec;40(12):2077-80. doi: 10.1016/j.joen.2014.09.009.

34. Libman H, Brockmeyer DM, Gold HS. Should we prescribe antibiotics to this patient with persistent upper respiratory symptoms?: Grand rounds discussion from Beth Israel Deaconess Medical Center. Ann Intern Med. 2017 Feb 7;166(3):201-208. doi: 10.7326/M16-2766.

35. MacDonald A, Newton CW. Pseudocyst of the maxillary sinus. J Endod. 1993 Dec;19(12):618-21. 